\title{
Experimental realization of phonon demultiplexing in three-dimensions
}

\author{
Osama R. Bilal ${ }^{1,2}$ and Chern Hwee Yee ${ }^{2}$, Jan Rys ${ }^{2}$, Christian Schumacher ${ }^{3}$, and Chiara Daraio ${ }^{4}$ \\ ${ }^{1}$ Department of Mechanical engineering, University of Connecticut, Storrs, USA \\ ${ }^{2}$ Department of Mechanical and Process engineering, ETH Zürich, 8092 Zürich, Switzerland \\ ${ }^{3}$ Computer Graphics Laboratory, ETH Zürich, 8092 Zürich, Switzerland and \\ ${ }^{4}$ Division of Engineering and Applied Science, California Institute of Technology, Pasadena, California 91125, USA
}

(Dated: September 28, 2020)

\begin{abstract}
Phononic metamaterials enabled the realization of many acoustic components analogous to their electronic counterparts, such as transistors, logic gates and calculators. A key component among these is the demultiplexer, a device that receives multiple signals and sorts them based on their frequencies into separate channels. Previous experimental realizations of acoustic and elastic multiplexers have employed plates with pillars or holes to demultiplex frequencies. However, existing realizations are confined to two-dimensions, which can limit potential acoustic or elastic circuit design. Here we show the first experimental realization of a three-dimensional, four channel phononic demultiplexer. Our design methodology is based on bundles of pass-bands within a large band gap that can easily be tuned for multi-channel frequency demultiplexing. The proposed design can be utilized in acoustic and elastic information processing, nondestructive evaluation and communication applications among others.
\end{abstract}

Phononic crystals and acoustic metamaterials consist of periodic arrangements of basic building blocks that repeat in space. These materials have the ability to control waves in an unprecedented manner, at different length and frequency scales. One of the unique properties of these materials is their ability to support forbidden frequency bands (i.e., band gaps) in their dispersion spectrum. Within a band gap, waves can not propagate and are reflected towards the source. The main mechanisms for creating these forbidden bands within the frequency spectrum are either destructive interference (e.g., in Bragg scattering band gaps) or local resonance. When a band gap is open due to Bragg scattering, the wavelength of the affected waves is usually at the same order of the spacing between the unit cells (i.e., lattice spatial periodicity) [1, 2]. In contrast, when a band gap is open due to resonances, the wavelength of the attenuated waves can be independent of the lattice spacing [3]. Such resonances induce properties that might not exist in conventional materials, like negative effective mass or stiffness [4-6]. Phononic crystals and metamaterials with such exotic properties have been utilized for many applications such as vibration and sound insulation [7, 8], seismic wave protection [9, 10], wave guiding [11, 12] and frequency filtering [13, 14] among others [15].

Phononic crystals and metamaterials have been proposed as platforms to enable mechanical information processing. Potential applications range from thermal computing [16[18] (at small scales) to ultrasound and acoustic based computing (at larger scales) [19, 20]. Current realizations of fundamental phonon computing elements such as acoustic switches [21, 22], rectifiers [23, 24], diodes [25,-28], transistors [29, 30], memory [31] and lasers [32, 33] have been inspired by their electronic counterparts. Among such devices, demultiplexers are a combinational logic devices that take a single input at one end and route it to one of several output channels (Fig. 11 1 ). There exist multiple theoretical proposals for realizing phonon demultiplexers for both acoustic [13, 34, 35] and elastic waves [36-44] with few experimental demonstrations [35, 45, 46]. The main working principle for most of these designs is based on embedded cylin- ders [13, 36, 44, 47], holes [45] or pillars [46] in a host plate. These realizations constrain demultiplexing phonons to twodimensions. A device that can demultiplex phonons in three dimensions for all wave polarization remains elusive. In this paper, we numerically design and experimentally characterize a 3D phonon demultiplexer (Fig. 1b).

To realize our demultiplexer, we design a cubic unit cell that has two important characteristics: (1) a wide band gap to filter out undesirable frequencies and (2) a bundle of pass bands inside the large band gap to allow only the targeted frequencies to pass. The unit cell is composed of eight masses, one at each a)

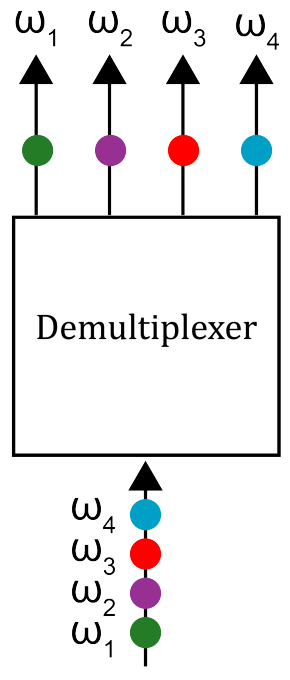

b)

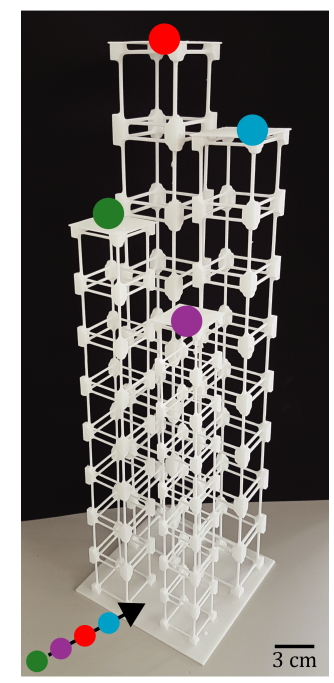

FIG. 1. 3D Phononic demultiplexer. a) A conceptual schematic of a 4-channel demultiplexer with four different input frequencies excited at one end and sorted in four predefined channels at the other end. b) A 3D printed realization of the 4-channel phonon demultiplexer, where four different frequencies are excited at the base and passively sorted into their corresponding channel. 
of the unit cell's corners, connected by 12 beams (Fig. 2a).

To verify our design hypothesis, we first consider an infinite unit cell model, where one single unit cell is assumed to repeat in space in all directions. We analyze the unit cell using Bloch periodic boundary conditions [48]. The dispersion curves of the unit cell, correlating wavenumber to frequency, are calculated using the wave equations for heterogeneous media [49] within an infinite medium. We use the finite element method to solve the elastic wave equations (COMSOL 5.4). The solution is the wavefunction $u(x, \kappa ; t)=\tilde{u}(x) \exp ^{\left(i\left(\kappa^{\top} x-\omega t\right)\right)}$, where $\tilde{u}$ is the Bloch displacement vector, $x$ is the position vector, $\kappa$ is the wavenumber, $\omega$ is frequency and $t$ is time. The resulting dispersion curves are normalized by the unit cell size and the speed of sound in the medium, $\Omega=f a / c$. The dispersion plot shows a significant band gap (shaded gray region in Fig. 2p) with relative width of $\approx 124 \%$. The relative width of the band gap is calculated as the absolute width of the band gap divided by its central frequency. Within the band gap region, there exist two pass bands with central frequency $\Omega=0.29 \& 0.53$, which we engineer to work as the transmis- a)

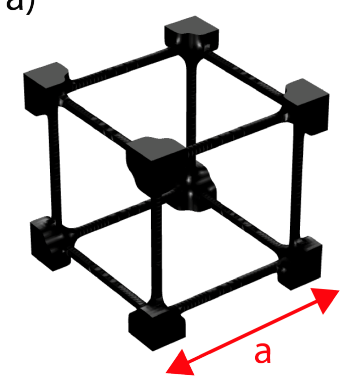

b)

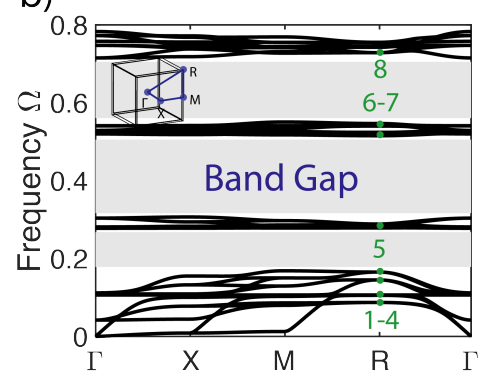

c)
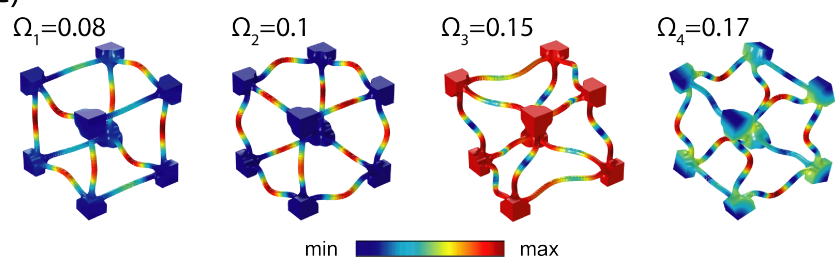

$\min$
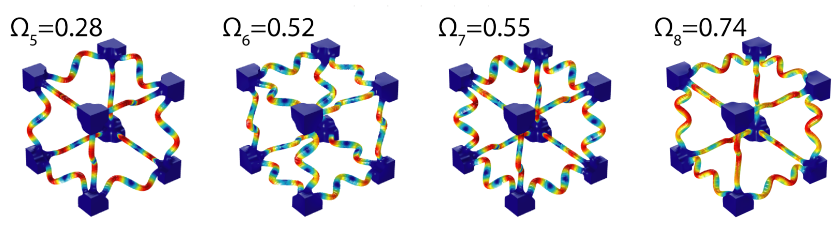

FIG. 2. Basic building block. a) The unit cell composed of eight masses at its corners connected with 12 beams forming a cube. b) Dispersion curves of the metamaterial unit cell along the path $\Gamma-X-M-R-\Gamma$ assuming infinite repetition of the unit cell in all directions. Full band gaps, where waves are prohibited from propagation, are highlighted in gray. The inset shows the irreducible Brillouin zone for a symmetric cubic unit cell. (c) Selected elastic mode shapes of the unit cell at the $R$ point at the four different transmission bands. sion bands for our demultiplexer.

To better explain the behavior of the proposed design, we visualize the unit-cell's vibrational mode shapes by superimposing the displacement profiles as a heat map over its geometry for eight different frequencies (Fig. (2))). The first two mode shapes $\left(\Omega_{1,2}=0.08 \& 0.1\right)$ resemble the first vibrational modes of the beams connecting the corner masses. The third and fourth modes $\left(\Omega_{3,4}=0.15 \& 0.17\right)$ show the mixed resonance modes of the corner masses along with the first vibrational modes of the connecting beams. All four modes exist below the the band gap. The fifth mode shape, which exists in the first pass band $\left(\Omega_{5}=0.28\right)$, shows the second vibrational mode of the connecting beams. The third vibrational mode of the unit cell beams manifests itself within the second pass band $\left(\Omega_{6,7}=0.52 \& 0.55\right)$. At the edge of the band gap $\left(\Omega_{8}=0.74\right)$, the connecting beams vibrate at their fourth mode shape. The masses work as pivots or fixation points for the connecting slender beams, which behave as fixed-fixed beams with well defined vibrational mode shapes. Based on this simple design concept, the unit cell can be considered as a simple mass spring model with a large band gap with embedded bands of transmission. By changing the diameter of the beams or the ratio between the mass of the beam to the mass of the corner block, we can change the position of the band gap and its width. Alternatively, by scaling the unit cell length $a$, we can shift the position of the band gap along with its embedded pass bands within the frequency spectrum. An advantage of scaling the entire unit cell while keeping all aspect ratios the same, is preserving all the dispersion characteristics of the unit cell (e.g., group velocities) without alteration. This preservation can help in maintaining consistency in the performance of the different channels, such as the mode shapes and wave polarization.

The demultiplexer is constructed by designing four channels out of the same unit cell, but with four different lattice constants. All four channels are connected at one end (i.e, the base of the demultiplexer). The excitation takes place at the base with multiple frequencies that get sorted into the four channels. To correctly confirm the behavior of the scaled unit cells, we calculate the dispersion curves for four different lattice constants $a=30,36,42$ and $48 \mathrm{~mm}$ (Fig. 3h,c,e and g). The dispersion curves for these four unit cells are calculated based on 1D Bloch periodicity (only along the $\kappa_{x}$ direction). Basing the analysis on 1D periodicity, as opposed to 3D periodicity, gives rise to extra modes along the free boundaries with no periodicity. These boundary modes manifest themselves as added lines in the dispersion curves. A clear difference between the $1 \mathrm{D}$ and $3 \mathrm{D}$ periodic dispersion curves can be seen, for example, by comparing the number of branches below the first band gap in Fig. $2 \mathrm{p}$ and 3 a. The $1 \mathrm{D}$ periodic dispersion curves serve as the limiting case for the performance of the proposed design. As more periodicity is incorporated in the model (i.e., along $\kappa_{y} \& \kappa_{z}$ direction), less vibration modes become available at the free boundaries and the dispersion curves become identical to figure $2 \mathrm{p}$. Therefore, having a demultiplexer channel with more unit cells in all directions will result in less dispersion lines and wider band gaps.

To validate the infinite unit cell model against the finite 

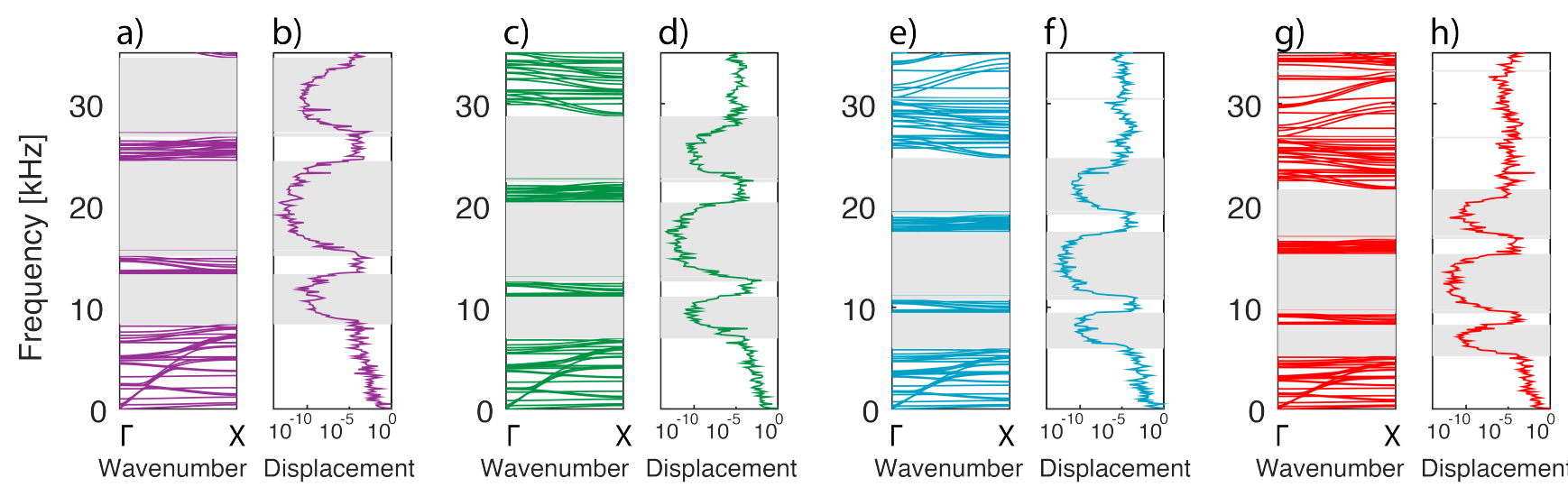

Wavenumber Displacement

Wavenumber Displacement

FIG. 3. Infinite medium vs finite structure. The calculated dispersion curves and their corresponding frequency response functions (FRF) for unit cells with side length equal to (a-b) $30 \mathrm{~mm}$ (c-d) $36 \mathrm{~mm}$ (e-f) $42 \mathrm{~mm}$ (g-h) $48 \mathrm{~mm}$. The dispersion curves are calculated along the $\Gamma-X$ direction with periodicity only along the $\mathrm{x}$ direction. The FRFs are calculated for a structure composed of $1 \times 1 \times 8$ unit cells. The band gaps are highlighted in gray in both dispersion curves and FRFs.

structure with all the connected channels, we numerically simulate the exact geometry of the demultiplexer as seen in figure 1 b using the finite element method. Each channel is composed of an array of 8 unit cells tessellated along the $\mathrm{z}$ direction. All channels are connected to a base plate with thickness $T_{h}=$ $3 \mathrm{~mm}$. The top end of each channel is terminated by a plate with the same thickness for signal extraction. The channels are arranged in a $2 \times 2$ grid separated by $30 \mathrm{~mm}$. A harmonic excitation is applied at the bottom surface of the base plate. We sweep the excitation frequency between 1 and 32 $\mathrm{kHz}$, and record the displacement at the end of each of the channels (Fig. 3b,d,f and h). The predicted band gap frequencies using the infinite unit cell model are shaded in gray in all panels of figure 3 . The results of both the finite and infinite models are in a very good agreement. Within the band gaps, shown as the gray shaded regions in figure 3 , the amplitude
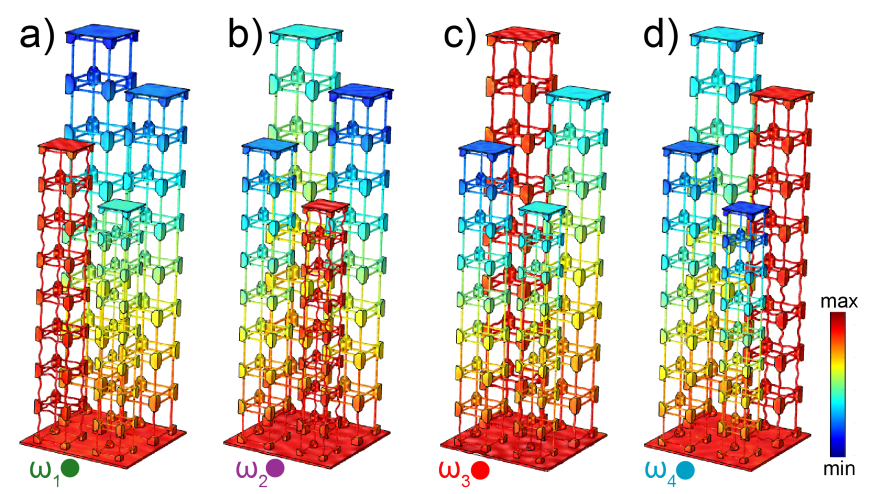

FIG. 4. Demultiplexer numerical simulations. Numerically calculated mode shapes of the demultiplexer operating at a) $\omega_{1}=11.7$ $\left.\mathrm{kHz}, \mathrm{b}) \omega_{2}=13.9 \mathrm{kHz}, \mathrm{c}\right) \omega_{3}=15.9 \mathrm{kHz}$ and d) $\omega_{4}=18.1 \mathrm{kHz}$. The heat map represents the intensity of motion (i.e., the logarithm of displacement) through the entire structure when excited at the base. of the transmitted wave is many orders of magnitude less than that of the pass band frequencies. The fact that all channels are connected at the base is not affecting the robustness of the individual channels' performance. In addition, we also plot the logarithm of the full displacement fields as a heat map at the four operational frequencies of the demultiplexer (Fig. 47. The displacement field at each frequency shows a clear transmission of elastic phonons at targeted frequency, while the rest of the channels show negligible motion.

To experimentally validate our numerical simulations, we fabricate the demultiplexer through additive manufacturing (laser sintering) using polyamide (Young's modulus $E=2.3$

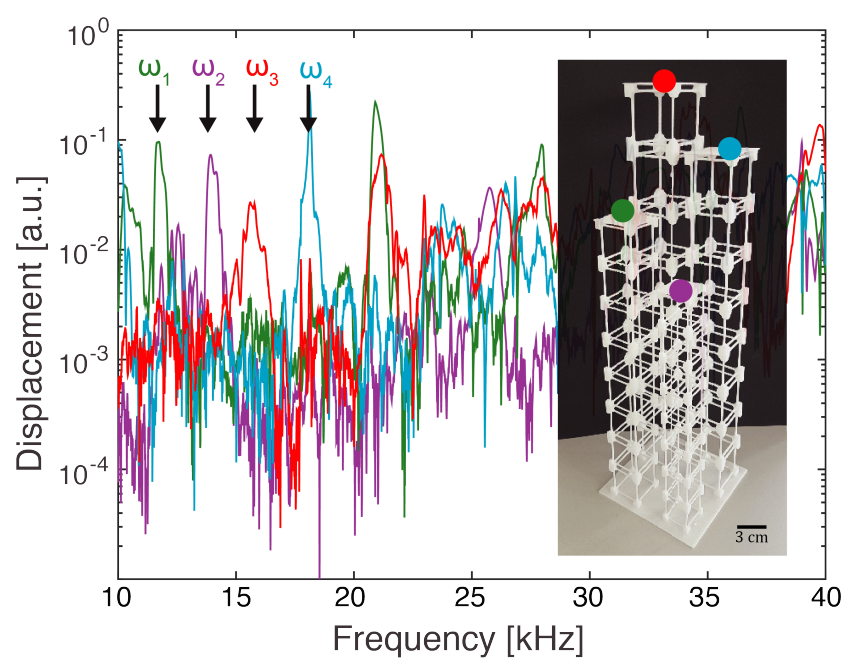

FIG. 5. Demultiplexer experimental characterization. Experimental measurements of the displacement at the end of each channel as a function of frequency. The targeted frequencies are marked with black arrows with $\omega_{1}=11.7 \mathrm{kHz}, \omega_{2}=13.9 \mathrm{kHz}, \omega_{3}=15.9 \mathrm{kHz}$ and $\omega_{4}=18.1 \mathrm{kHz}$. 
GPa and density $\rho=1200 \mathrm{Kg} / \mathrm{m}^{3}$ ). We characterize the vibration response of the meta-device by harmonically exciting the base plate with a piezoelectric disc (repeating the previously performed numerical simulations in figure 3, 4). We measure the transmitted vibrations at the end of each channel with a laser Doppler vibrometer LDV (Polytec OFV-505 with a OFV-5000 decoder, using a VD-06 decoder card). We excite the system by sweeping through frequencies between 10 and $40 \mathrm{kHz}$ and record the amplitude of the transmitted vibrations at the end of each channel (Fig. 5). The measured vibrations between 10 and $20 \mathrm{kHz}$ show a clear band gap, as predicted by the numerical simulations in all channels, and demonstrate clearly transmission of the signal in the designated pass bands in each channel. The experimental measurements show a clear evidence of phonon demultiplexing based on frequency in the realized meta-device.

In this study, we design, analyze and realize the first 3D phononic demultiplexer. Our design relies on defining relatively narrow pass bands within a wide band gap. Design- ing the width of the pass band allows for a wider operational bandwidth and ensures transmission of each signal to its designated channel. The complete band gap for all directions and polarization (in-plane, out of plane and rotation) of the wave allows for phonon demultiplexing in three-dimensions. Multiplexing elastic and acoustic waves in 3D can enable the design of higher dimensional all-phononic circuits taking an advantage of the recent progress in advanced manufacturing. Due to these advances in fabrication and the material invariant nature of the design principle, our meta-device can be easily scaled to operate at desired frequencies with no geometry alteration. For example, the same design fabricated at half the size would operate around twice the frequency (i.e., in the ultrasound regime). Our demultiplexer has possible applications in imaging, communications and phonon based information processing.

The authors are very grateful for the fruitful discussions with Dr. Foehr and his help with the experimental setup.
[1] M. Kushwaha, P. Halevi, L. Dobrzynski, and B. DjafariRouhani, Acoustic band structure of periodic elastic composites, Physical Review Letters 71, 2022 (1993).

[2] M. Sigalas and E. Economou, Band structure of elastic waves in two dimensional systems, Solid State Communications 86, 141 (1993).

[3] Z. Liu, X. Zhang, Y. Mao, Y. Zhu, Z. Yang, C. Chan, and P. Sheng, Locally resonant sonic materials, Science 289, 1734 (2000).

[4] J. Christensen, M. Kadic, O. Kraft, and M. Wegener, Vibrant times for mechanical metamaterials, Mrs Communications 5, 453 (2015).

[5] S. A. Cummer, J. Christensen, and A. Alù, Controlling sound with acoustic metamaterials, Nature Reviews Materials 1, 16001 (2016).

[6] G. Ma and P. Sheng, Acoustic metamaterials: From local resonances to broad horizons, Science advances 2, e1501595 (2016).

[7] Z. Yang, H. Dai, N. Chan, G. Ma, and P. Sheng, Acoustic metamaterial panels for sound attenuation in the 50-1000 hz regime, Applied Physics Letters 96, 041906 (2010).

[8] O. R. Bilal, D. Ballagi, and C. Daraio, Architected lattices for simultaneous broadband attenuation of airborne sound and mechanical vibrations in all directions, Physical Review Applied 10, 054060 (2018).

[9] S.-H. Kim and M. P. Das, Seismic waveguide of metamaterials, Modern Physics Letters B 26, 1250105 (2012).

[10] S. Brûlé, E. Javelaud, S. Enoch, and S. Guenneau, Experiments on seismic metamaterials: Molding surface waves, Physical review letters 112, 133901 (2014).

[11] M. Torres, F. Montero de Espinosa, D. Garcia-Pablos, and N. Garcia, Sonic band gaps in finite elastic media: Surface states and localization phenomena in linear and point defects, Physical Review Letters 82, 3054 (1999).

[12] C. J. Rupp, A. Evgrafov, K. Maute, and M. L. Dunn, Design of phononic materials/structures for surface wave devices using topology optimization, Structural and Multidisciplinary Optimization 34, 111 (2007).

[13] Y. Pennec, B. Djafari-Rouhani, J. Vasseur, A. Khelif, and P. A.
Deymier, Tunable filtering and demultiplexing in phononic crystals with hollow cylinders, Physical Review E 69, 046608 (2004).

[14] C. J. Rupp, M. L. Dunn, and K. Maute, Switchable phononic wave filtering, guiding, harvesting, and actuating in polarization-patterned piezoelectric solids, Applied Physics Letters 96, 111902 (2010).

[15] M. Maldovan, Sound and heat revolutions in phononics, Nature 503, 209 (2013).

[16] B. Li, L. Wang, and G. Casati, Negative differential thermal resistance and thermal transistor, Applied Physics Letters 88, 143501 (2006).

[17] L. Wang and B. Li, Thermal logic gates: computation with phonons, Physical review letters 99, 177208 (2007).

[18] K. Joulain, J. Drevillon, Y. Ezzahri, and J. Ordonez-Miranda, Quantum thermal transistor, Physical review letters 116, 200601 (2016).

[19] S. Bringuier, N. Swinteck, J. Vasseur, J.-F. Robillard, K. Runge, K. Muralidharan, and P. Deymier, Phase-controlling phononic crystals: Realization of acoustic boolean logic gates, The Journal of the Acoustical Society of America 130, 1919 (2011).

[20] T. Zhang, Y. Cheng, J.-z. Guo, J.-y. Xu, and X.-j. Liu, Acoustic logic gates and boolean operation based on self-collimating acoustic beams, Applied Physics Letters 106, 113503 (2015).

[21] S. Babaee, N. Viard, P. Wang, N. X. Fang, and K. Bertoldi, Harnessing deformation to switch on and off the propagation of sound, Advanced Materials (2015).

[22] O. R. Bilal, A. Foehr, and C. Daraio, Reprogrammable phononic metasurfaces, Advanced materials 29, 1700628 (2017).

[23] B. Liang, B. Yuan, and J.-c. Cheng, Acoustic diode: rectification of acoustic energy flux in one-dimensional systems, Physical review letters 103, 104301 (2009).

[24] B. Liang, X. Guo, J. Tu, D. Zhang, and J. Cheng, An acoustic rectifier, Nature materials $\mathbf{9}, 989$ (2010).

[25] B.-I. Popa and S. A. Cummer, Non-reciprocal and highly nonlinear active acoustic metamaterials, Nature communications 5 (2014).

[26] X.-F. Li, X. Ni, L. Feng, M.-H. Lu, C. He, and Y.-F. Chen, Tun- 
able unidirectional sound propagation through a sonic-crystalbased acoustic diode, Physical review letters 106, 084301 (2011).

[27] T. Devaux, V. Tournat, O. Richoux, and V. Pagneux, Asymmetric acoustic propagation of wave packets via the selfdemodulation effect, Physical review letters 115, 234301 (2015).

[28] N. Boechler, G. Theocharis, and C. Daraio, Bifurcation-based acoustic switching and rectification, Nature materials 10, 665 (2011).

[29] D. Hatanaka, I. Mahboob, K. Onomitsu, and H. Yamaguchi, A phonon transistor in an electromechanical resonator array, Applied Physics Letters 102, 213102 (2013).

[30] O. R. Bilal, A. Foehr, and C. Daraio, Bistable metamaterial for switching and cascading elastic vibrations, Proceedings of the National Academy of Sciences 114, 4603 (2017).

[31] D. Hatanaka, I. Mahboob, K. Onomitsu, and H. Yamaguchi, Mechanical random access memory in a phonon circuit, Applied Physics Express 7, 125201 (2014).

[32] K. Vahala, M. Herrmann, S. Knünz, V. Batteiger, G. Saathoff, T. Hänsch, and T. Udem, A phonon laser, Nature Physics 5, 682 (2009).

[33] H. Jing, S. Özdemir, X.-Y. Lü, J. Zhang, L. Yang, and F. Nori, Pt-symmetric phonon laser, Physical review letters 113, 053604 (2014).

[34] B. Rostami-Dogolsara, M. K. Moravvej-Farshi, and F. Nazari, Designing switchable phononic crystal-based acoustic demultiplexer, IEEE transactions on ultrasonics, ferroelectrics, and frequency control 63, 1468 (2016).

[35] A. A. Watkins and O. R. Bilal, Demultiplexing infrasound phonons with tunable magnetic lattices (2020), arXiv:2009.08381 [physics.app-ph]

[36] Q. Zou, W. Liu, T. Yu, N. Liu, T. Wang, and Q. Liao, Decoupling of multiple coupled phononic crystal waveguides: application to acoustic demultiplexing, Journal of Physics D: Applied Physics 50, 125102 (2017).

[37] P. Moradi and A. Bahrami, Three channel ghz-ranged demultiplexer in solid-solid phononic crystals, Chinese Journal of Physics 59, 291 (2019).
[38] F. Motaei and A. Bahrami, Eight-channel acoustic demultiplexer based on solid-fluid phononic crystals with hollow cylinders, Photonics and Nanostructures-Fundamentals and Applications 39, 100765 (2020).

[39] F. Nazari and J. Babaki, Heterostructure based demultiplexer using solid-solid phononic crystal ring resonators, Journal of Physics D: Applied Physics (2020).

[40] Y. Ben-Ali, A. Khaled, D. Bria, et al., Y-shaped branch structure using asymmetric resonators for phononic demultiplexing, Materials Today: Proceedings (2020).

[41] H. Gharibi and A. Bahrami, Phononic crystals for sensing fames with demultiplexed frequencies, Journal of Molecular Liquids , 112841 (2020).

[42] Y. Pennec, B. Djafari-Rouhani, J. Vasseur, H. Larabi, A. Khelif, A. Choujaa, S. Benchabane, and V. Laude, Channel drop process of elastic wave in a two dimensional phononic crystal, in IEEE Ultrasonics Symposium, 2005., Vol. 1 (IEEE, 2005) pp. 69-72.

[43] M. I. Hussein, G. M. Hulbert, and R. A. Scott, Hierarchical design of phononic materials and structures, in ASME International Mechanical Engineering Congress and Exposition, Vol. 42258 (2005) pp. 163-172.

[44] J. Vasseur, O. B. Matar, J. Robillard, A.-C. Hladky-Hennion, and P. A. Deymier, Band structures tunability of bulk $2 \mathrm{~d}$ phononic crystals made of magneto-elastic materials, AIP advances 1, 041904 (2011).

[45] S. Mohammadi and A. Adibi, On chip complex signal processing devices using coupled phononic crystal slab resonators and waveguides, AIP Advances 1, 041903 (2011).

[46] M. S. Faiz, M. Addouche, A. R. M. Zain, K. S. Siow, A. Chaalane, and A. Khelif, Experimental demonstration of a multichannel elastic wave filter in a phononic crystal slab, Applied Sciences 10, 4594 (2020).

[47] B. Rostami-Dogolsara, M. K. Moravvej-Farshi, and F. Nazari, Acoustic add-drop filters based on phononic crystal ring resonators, Physical Review B 93, 014304 (2016).

[48] F. Bloch, "U about the quantum mechanics of electrons in crystal lattices, Journal of Physics 52, 555 (1929).

[49] K. F. Graff, Wave motion in elastic solids (Courier Corporation, 2012). 